\title{
No link between epidurals and autism, two studies confirm
}

\author{
BY LAURA DATTARO
}

28 SEPTEMBER 2021

\section{Listen to this story:}

https://www.spectrumnews.org/wp-

content/uploads/2021/09/audio-4bb7910a-9d9b-4053-bb34-ba440e3803f5-encodings.mp3

The epidural anesthesia commonly given to pregnant people during labor is not linked to autism in children, according to two studies published today in the Journal of the American Medical Association.

The results contradict a study published last October that seemingly connected epidurals among women in California to a slightly increased chance of autism in their children. That study drew widespread criticism and concern from researchers and professional medical societies for its failure to account for confounding factors, such as a family history of psychiatric conditions. In April, a follow-up study in Manitoba, Canada, that controlled for some additional factors found no such association.

"There really is no solid evidence, from any of the studies actually, that epidural labor analgesia causes autism," says Cynthia Wong, professor of anesthesia at the University of lowa in lowa City, who co-wrote a commentary accompanying the new studies. "I don't think either clinicians or parents at this point in time need to take that consideration into account."

The two new studies probe the connection further, using different populations and statistical methods. In one, researchers examined the health records of 388,254 children born in British Columbia, Canada, from 2000 to 2014. Because British Columbia has a central autism assessment network, the majority of children in the study went through the same screening and diagnostic process, which was not true for participants in the earlier studies.

About 1.5 percent of the Canadian children exposed to an epidural went on to be diagnosed with 


\section{Spectrum | Autism Research News}

https://www.spectrumnews.org

autism, compared with 1.3 percent of unexposed children, suggesting a small association.

But further analyses of the data weakened the finding. To control for some factors, such as maternal genetics and socioeconomic status, for example, the researchers repeated the analysis in women who had multiple births, resulting in at least one child with autism and one without. The women were not more likely to have had an epidural while delivering the autistic child than the nonautistic one, they found.

\section{Making adjustments:}

In another study, researchers analyzed data from 479,178 children born in Denmark from 2006 through 2013. Unlike the other three studies, this analysis controlled for family history of autism and maternal psychiatric history, both of which are linked to an increased likelihood of autism. The researchers found no significant association between autism and epidurals.

"Assessing this link provides an excellent example of why confounders are almost always essential to address in an observational study," says Anders Mikkelsen, an obstetrician and doctoral student at Copenhagen University Hospital - Rigshospitalet in Denmark and lead investigator on the Danish study. "Personally, I would not have reservations about recommending a labor epidural to patients or family members seeking advice on labor pain management."

All four studies also identified differences between parents who did and did not receive an epidural. For example, people who received an epidural tended to be younger and were more likely to have preeclampsia than those who didn't - both factors linked to autism. And women who received an epidural were more likely to have a psychiatric condition, and their children were more likely to have a parent with autism. The differences suggest that none of the studies fully eliminated confounding factors, Wong wrote in the commentary.

"This is very complicated observational research to do," says Gillian Hanley, assistant professor of obstetrics and gynecology at the University of British Columbia and lead investigator on the new Canadian study. "Having multiple people do it, and do it slightly differently, and do it in different populations, and do it with different data, is valuable in the long run for getting us to the right answer to this question."

Together, the studies are "reassuring" that epidurals are not associated with autism, Hanley says. Still, she says, researchers should attempt to work out which confounding factors lead to both use of epidural anesthesia and increased rates of autism diagnoses.

"We owe it to women to try to sort this out," Hanley says.

Cite this article: https://doi.org/10.53053/EGSO3396 